\title{
Bridging the imaging gap in nanobiology with three-dimensional electron microscopy
}

Sriram Subramaniam*

*Center for Cancer Research, National Cancer Institute, Bethesda, MD 20892

Emerging methods in three-dimensional biological electron microscopy provide powerful tools and great promise to bridge a critical gap in imaging in the biomedical size spectrum. This gap comprises a size range of great interest in biology and medicine that includes cellular protein machines, giant protein and nucleic acid assemblies, small subcellular organelles and small bacteria. These objects are generally too large and/or too heterogeneous to be investigated by high resolution X-ray and NMR methods; yet the level of detail afforded by conventional light and electron microscopy is often not adequate to describe their structures at resolutions high enough to be useful in understanding the chemical basis of biological function.

In our laboratory at the National Cancer Institute, $\mathrm{NIH}$, we are using a variety of approaches that utilize electron microscopic imaging to discover and analyze biological complexity within the size gap with linear dimensions of about $50-1000 \mathrm{~nm}$. Ultimately, the understanding of cellular architecture gained at this level will be crucial in designing effective strategies for disease prevention and treatment. A key mission of our laboratory is to quantitatively describe the spatial and temporal architecture of key molecular machines that fall into this "nano gap". Areas of current interest include: (i) the development and application of novel technologies for three-dimensional electron microscopy of specimens ranging in size from small molecules to tissues, including automated approaches to analyze the molecular structure and sub-cellular location of a variety of nanoparticles, (ii) determination of the dynamic spatial and temporal architectures of cellular structures and molecular machines involved in fundamental process such as energy transduction, cell division and chemotaxis, and (iii) determination of molecular mechanisms underlying the neutralization and cellular entry of HIV.

Subramaniam, S. and Milne, J.L.S. (2004) Three-dimensional electron microscopy at molecular resolution Ann. Rev. Biophys. Biomol. Struct. 33, 141-155.

Subramaniam, S. (2005) Bridging the imaging gap: Visualizing subcellular architecture with electron tomography Curr. Opin. Microbiology $\underline{8}$, 316-322. 


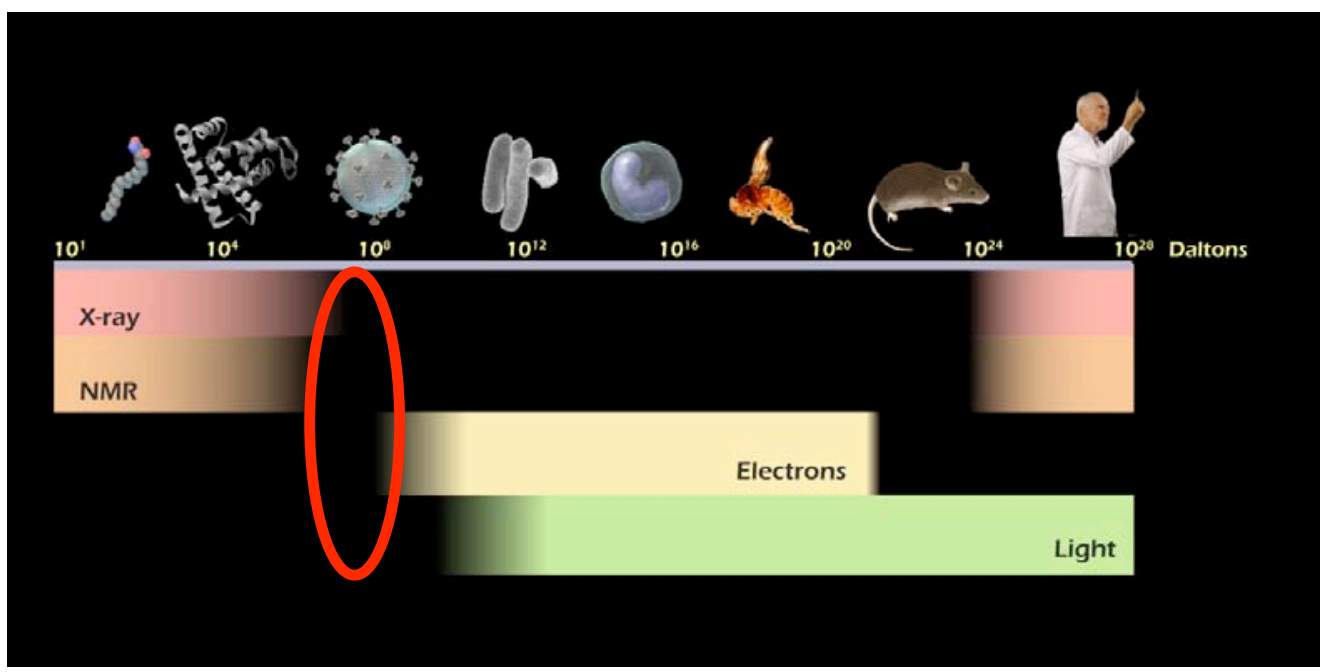

Fig. 1: Bridging the nano gap: Imaging technologies based on X-rays, magnetic resonance, light and conventional electron microscopy span most of the size range of interest in biology. However, a small size range, indicated by the red oval is not easily accessible by these methods. Emerging methods in 3D electron microscopy offer a unique opportunity to bridge this gap in imaging objects in the "nano" size range (from ref $1)$.

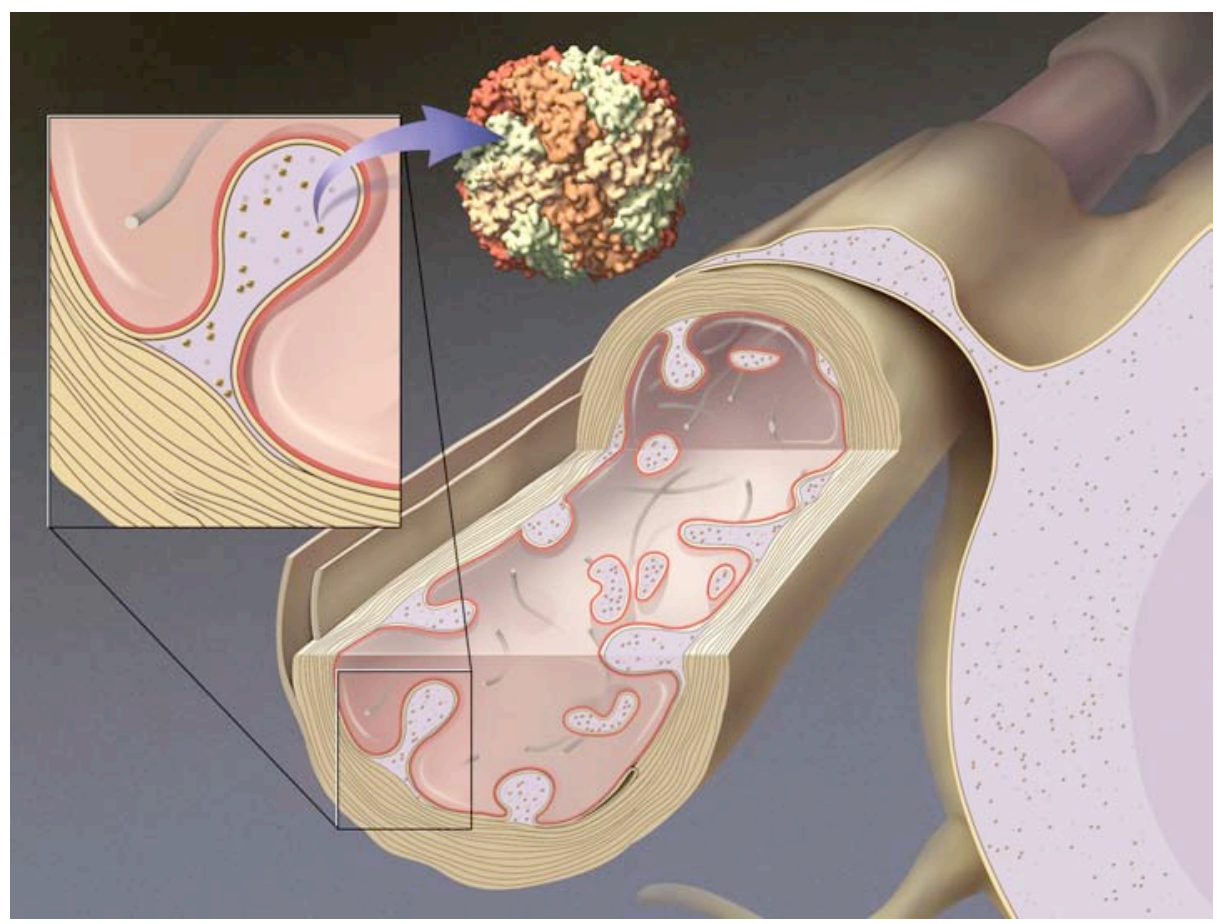

Fig. 2: 3D localization of ferritin in degenerating neurons using electron tomography. Artistic rendering of the cellular anatomy of axonal degeneration in transgenic mice carrying knockouts for genes for the two iron regulatory proteins IRP1 and IRP2. The brown dots represent ferritin particles, whose molecular structure is shown at the head of the blue arrow. Electron tomographic studies reveal that most of the excess ferritin observed in these degenerating axons is localized to regions in the interior that arise from invaginations of neighboring oligodendrocyte cells (shown at lower right) into the axon. (from Zhang et al J. Struct. Biol. 150, 144-153 (2005). 